\title{
Surface Premelting and Interfacial Interactions of Semi-Clathrate Hydrate
}

\author{
Ngoc N. Nguyen, ${ }^{*},+\ddagger$ Rüdiger Berger, ${ }^{\dagger}$ and Hans-Jürgen Butt ${ }^{*}{ }^{\dagger}$ (i)
}

${ }^{\dagger}$ Department of Physics at Interfaces, Max Planck Institute for Polymer Research, Ackermannweg 10, 55128 Mainz, Germany

${ }^{\ddagger}$ School of Chemical Engineering, Hanoi University of Science and Technology, Dai Co Viet Street 1, 100000 Hanoi, Vietnam

\section{Supporting Information}

ABSTRACT: Clathrate hydrates are a demanding field of research because of their fundamental impact on human life and the environment. Interfacial forces involving hydrates are vital factors in numerous natural and industrial processes, but the topic has been relatively neglected in the scientific literature. By using atomic force microscopy, we are now able to measure for the first time the forces between a semiclathrate hydrate formed by tetrabutyl ammonium bromide and a silica microsphere in air and in undecane. Significantly, the analyses of jump-in (attractive) and pull-off (adhesive) forces in force-distance curves indicate the presence of a quasi-liquid layer (QLL) on the hydrate surface resulting from interfacial premelting. We have shown

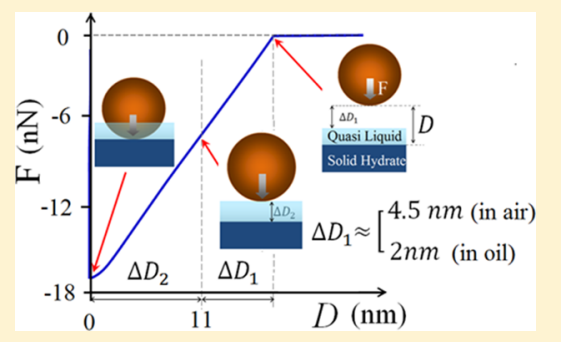
that interfacial forces between the sphere and the hydrate surface are dominated by the capillary formation between the QLL and the sphere. van der Waals forces are still active in the region several $\mathrm{nm}$ above the QLL, prior to the sphere touching the QLL. Our study introduces a new quantitative parameter to the hydrate literature, that is, the thickness of the QLL on the hydrate surface. This parameter, being around $11 \mathrm{~nm}$ at $-4{ }^{\circ} \mathrm{C}$, is crucial to the modeling of hydrate interactions and agglomerations.

\section{INTRODUCTION}

Clathrate hydrates, or hydrates, are solid solutions of water and hydrophobic guests. The water molecules form ice-like crystalline structures into which guest molecules are incorporated. ${ }^{1}$ Many substances (guests) can form hydrate structures with water, including gases $\left(\mathrm{CH}_{4}, \mathrm{CO}_{2}, \mathrm{~N}_{2}, \mathrm{H}_{2}\right.$, etc.), liquid hydrocarbons (cyclopentane- $\mathrm{CP}$ ) and water-miscible organics (tetrahydrofuran-THF and tetrabutyl ammonium bromide-TBAB). ${ }^{2}$ All known hydrates form at temperatures of a few degree celsius and various guest-dependent pressures, which range from ambient pressure for $\mathrm{CP}$, THF, and TBAB hydrates, up to a hundred bars and higher for gas hydrates. Hydrates are becoming increasingly significant because of their abundance in nature, relevance to industrial applications and importance for understanding water-guest hydrophobic interactions. ${ }^{3}$ In nature, methane hydrates are found abundantly on seafloors and in permafrost regions. ${ }^{4,5}$ Methane hydrates may contribute considerably to future energy and hydrocarbon supply. ${ }^{4,5}$ Moreover, hydrates are thought to be plentiful on certain other planets. ${ }^{6}$ In industry, however, hydrates can be a negative factor impeding the safety and efficiency of production. For example, they are the major cause of blockage of oil and gas pipelines where they form with residual water. ${ }^{7,8}$ Even so, as hydrates contain huge contents of guests and their formation/dissociation is completely reversible by tuning the prevailing conditions, they also offer innovative pathways for storage and separation of gases, ${ }^{9-16}$ sequestration of carbon dioxide, ${ }^{17,18}$ eco-friendly refrigeration, ${ }^{19,20}$ and water desalination. $^{21,22}$
Interfacial forces involving hydrate surfaces (IFHS) are of profound importance in nature and engineering. In general, interfacial force is a term to indicate the forces between two interfaces. In this paper, we define the term "IFHS" as the forces acting between a hydrate surface and another surface or particle, for example, the forces acting between a TBAB semiclathrate hydrate surface and a silica microsphere. In nature, IFHS are vital to interactions between natural gas hydrates and the surrounding environment. Although there are no previous studies on interactions involving hydrates in ocean sediments or permafrost regions, we can assume, based on general physical and chemical principles, that the surface of hydrate deposits therein must equilibrate mechanically, physically, and chemically with the surrounding environments. Otherwise they will dissociate and the gas will escape from the hydrate deposits. Right now this effect is negligible. Interfacial forces in general include electrostatic interactions (e.g., electric doublelayer forces), van der Waals (vdW) interactions, hydration forces, capillary forces, intermolecular interactions and hydrogen-bonding, and so forth. ${ }^{23}$ Therefore, IFHS must play important roles in governing the stability of hydrate surfaces and the interactions therein with minerals and biomasses.

In industry, IFHS govern, for example, the formation of hydrate-plugs of flow lines. ${ }^{8}$ Hydrate formation occurs naturally in subsea pipelines where all the prerequisite conditions of hydrate formation are met, that is, low

Received: July 4, 2019

Revised: August 21, 2019

Published: August 30, 2019 
temperature, high hydrostatic pressure, and water-guest coexistence. Dispersed water droplets form hydrate particles with hydrocarbons. The initial state of the resulting hydrate particles suspended in oil fluids is harmless because these particles can be transported safely with the flow. However, the suspended hydrates, as driven by IFHS, tend to aggregate during transportation, deposit and build up on the wall of the pipe and eventually block the flow. The problem is becoming more serious as offshore drilling moves to deeper water where hydrates form under higher driving forces. ${ }^{4}$ There is now an urgent need for scientists to develop new and more effective flow assurance strategies. A promising strategy is to use antiagglomerants (AAs) to prevent the formation of hydrate aggregates. $^{24-26}$ As surface-active agents, the AAs adsorb on the hydrate surface, alter the interfacial properties, and hinder hydrate aggregation. However, the target development of AAbased flow assurance strategy demands insights into IFHSthe driving force of hydrate agglomeration.

Because of the vital importance of IFHS, there have been significant efforts to quantify them. ${ }^{27-32}$ However, previous studies have been limited to measuring the detaching force between hydrate particles, or between hydrate particles and solid surfaces using a mechanical force apparatus (MFA). ${ }^{27-31}$ These measurements revealed detaching forces in the order of $10^{-3} \mathrm{~N} / \mathrm{m} \cdot{ }^{27-31}$ By using the MFA, only the force required to separate the two objects is measured and such a detaching force provides limited information about interfacial interactions involving hydrate surfaces. IFHS, which act between interfaces prior to attachment, have never been actually measured even though these forces govern the ability to form attachments. Gaining in-depth knowledge of IFHS is of fundamental importance for thoroughly understanding hydrate aggregation.

In this study we report on the direct measurement of IFHS for the first time using atomic force microscopy (AFM). Using a special setup as described in the Experimental Method section, we were able to achieve a stable low temperature environment ranging from 0 to $-5{ }^{\circ} \mathrm{C}$, which was required for hydrate stability. In addition, we performed measurements under dry conditions in order to prevent water condensation on surfaces. Both the above conditions are critical for the success of the measurement (Figure S1, Supporting Information). We chose a $\mathrm{TBAB}$ semi-clathrate hydrate for our investigation as it is easy to prepare compared to other hydrates which require agitation and/or pressurization conditions for formation. ${ }^{15,16,19}$ TBAB semi-clathrate hydrate forms via crystallization in quiescent solutions under atmospheric pressure and a temperature below its equilibrium dissociation point $\left(T_{\mathrm{e}} \approx 9{ }^{\circ} \mathrm{C}\right)$. $^{33}$ Other compounds such as THF and CP also form hydrates at atmospheric pressures. However, these hydrates have lower equilibrium dissociation temperatures, that is, around 4.0 and $7.7^{\circ} \mathrm{C}$, respectively. ${ }^{33,34}$ Therefore, lower experimental temperature and thus more cooling are required. Moreover, CP is not water-miscible so that agitation is required for sample preparations. Therefore, we chose TBAB for this study. Certainly, improved understanding on TBAB semi-clathrate hydrate can explain a lot of the science related to clathrate hydrates in general. Hereafter, we will omit the term "semi-clathrate" and simply refer to "TBAB hydrate" or "hydrate", but will always mean "TBAB semi-clathrate hydrate". We crystallized pellets of the hydrate with a diameter of about $10 \mathrm{~mm}$ and a thickness of about 0.5 $\mathrm{mm}$ in sample containers. The samples were used for surface force measurements at a temperature of $T=-4{ }^{\circ} \mathrm{C}$, in either air or undecane oil. Subcooling of $\Delta T=T_{\mathrm{e}}-T=13{ }^{\circ} \mathrm{C}$ was applied in every measurement unless otherwise stated. We measured IFHS in undecane and in air because these fluids mimic the conditions of hydrates in oil and natural gas pipelines, respectively.

\section{EXPERIMENTAL METHOD}

We employed an atomic force microscope for surface force measurements (Bruker MultiMode 3a, equipped with a Peltier cooling element). During measurement, the temperature $(T)$ was kept below the equilibrium dissociation point of the hydrate $\left(T_{\mathrm{e}}\right)$ to prevent melting. To avoid condensation of water from the humid air on the surfaces, our home-built setup provided cooling and dehumidification simultaneously (Figure 1 ). It consisted of two parallel, component cooling systems.
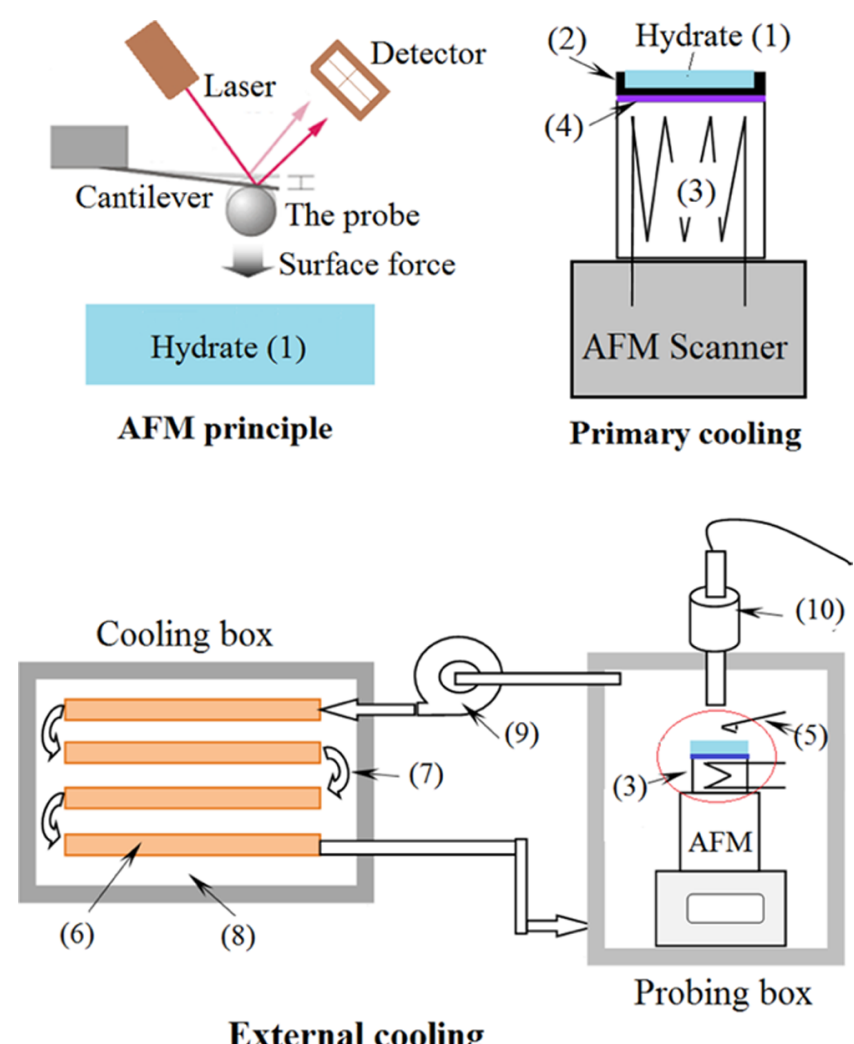

Figure 1. Schematics of the experimental setup for measurement of TBAB hydrate surface force, including hydrate (1), sample container (2), primary cooling based on the Peltier element (3), conductive paste (4), cantilever (5), heat-exchange tubes (6), U-type connecting tubes (7), dry-ice (8), air pump (9), and microscope camera (10). Other accessories are left out for simplicity.

Primary cooling was carried out with a commercial Peltier element (TAC Heater/Cooler, Bruker). This device could cool to a set-point down to $-7{ }^{\circ} \mathrm{C}$ with an accuracy of $0.1{ }^{\circ} \mathrm{C}$. The temperature was controlled by a digital controller (not shown). The upper surface of the Peltier element contacted thermally with a copper sample container (2) through a thin layer of conductive paste (4). However, only cooling the sample by using the Peltier element proved to be inadequate as water tended to condense from the air onto the hydrate surface (Figure S1, Supporting Information). Therefore, the atomic force microscope was then placed into an insulation box. The 
internal air was circulated through heat-exchange tubes, cooling the air to around $-30{ }^{\circ} \mathrm{C}$ inside the tubes indirectly by using dry-ice ( solid $\mathrm{CO}_{2},-78^{\circ} \mathrm{C}$ ) outside the tubes. At this low temperature, the humidity of the air was removed via frost deposition on the tubes surface (Figure S1, Supporting Information). By adjusting the circulation flow, we were able to maintain and stabilize the temperature in the insulation box at a desired value ranging between 0 and $-7{ }^{\circ} \mathrm{C}$. Note that primary cooling was still necessary in order to avoid heating of the sample by the AFM scanner and nearby electronics.

The TBAB hydrate was prepared via crystallization in a solution of stoichiometric composition $(1 \mathrm{~mol} \mathrm{TBAB}$ and 26 mol $\mathrm{H}_{2} \mathrm{O}$, or $40 \%$ by mass). ${ }^{33,35}$ The setup for sample preparation is shown in Figure S2, Supporting Information. The sample container was completely filled with the solution and kept at $0{ }^{\circ} \mathrm{C}$ in an ice-melting bath. The container was covered by a glass slide to prevent evaporation and/or condensation. Crystallization at $0{ }^{\circ} \mathrm{C}$ prevented the formation of ice in the hydrate sample. Hydrate nucleation is a stochastic event and thus the time required for hydrate formation varied greatly from hours to days. ${ }^{36,37}$ In most cases, hydrate formation was completed after $10 \mathrm{~h}$. After hydrate formation, the sample was transferred onto the AFM stage at $-4{ }^{\circ} \mathrm{C}$. This temperature was maintained for all measurements unless otherwise stated.

Silica spheres (diameter $5 \mu \mathrm{m}$ ) were glued to tipless, rectangular cantilevers (NSC36, $L \times W \times T=110 \mu \mathrm{m} \times 32$ $\mu \mathrm{m} \times 1 \mu \mathrm{m}$, resonance frequency $90 \mathrm{kHz}$ ) under a microscope. ${ }^{38}$ We used a commercial fluid cell (Bruker) for measurements in undecane. Force experiments were performed using a MultiMode AFM instrument (Bruker) with a scan rate of $1 \mathrm{~Hz}$. Force-versus-height graphs were converted to force-versus-distance curves-briefly called "forces curves"-by subtracting the cantilever deflection from the height position of the sample. Cantilevers were calibrated using the built-in "thermal tune" function of the AFM instrument. Two cantilevers were used, with a spring constant of 0.60 and $1.19 \mathrm{~N} / \mathrm{m}$, respectively. Consumable materials used in this work included TBAB (Sigma-Aldrich, 99.5\%), undecane oil (Sigma-Aldrich, 99\%), silica beads (Bangs Laboratories, Inc.). De-ionised water used in this work was produced by a Milli- $Q$ purification system (Millipore, USA).

\section{RESULTS AND DISCUSSION}

Figure 2 shows a typical force curve of a TBAB hydrate surface interacting with a $5 \mu \mathrm{m}$ silica sphere in undecane. The blue line indicates the force as the sphere approaches the hydrate. The force between the sample and the sphere is practically zero at distances $>20 \mathrm{~nm}$, for example, at position (1). At distances $<20 \mathrm{~nm}$ the sphere feels an attractive force resulting in a jump to the surface at (2). This jump to the surface happens when the force gradient acting on the sphere starts to exceed the spring constant of the cantilever. The force at (2) is called jump-in force. The continuing approach of the cantilever holder after jump-to-contact leads to an upward bending of the cantilever at (3) which is equivalent to a repulsive force. The red line represents the force while retracting the cantilever holder from the surface. The minimum force attained at position (4) is called pull-off, or adhesive force. We observed no interaction for distances $>20 \mathrm{~nm}$ in either undecane or in air, meaning that long-range electrostatic forces were absent, indicating that the hydrate surface was not charged. Force curves measured in undecane and in air showed similar
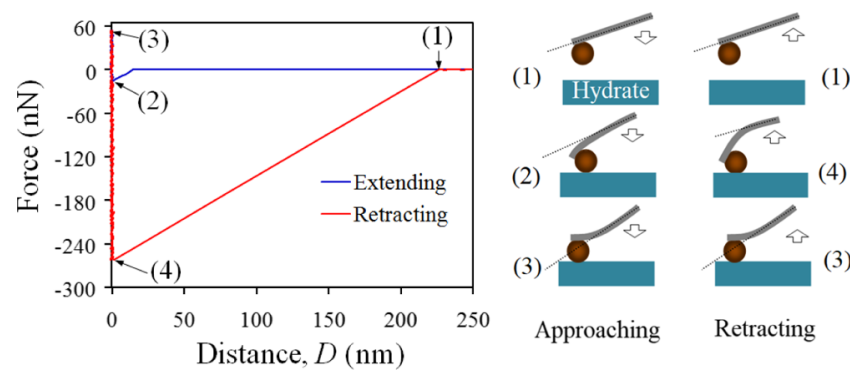

Figure 2. Left: typical force curve of a TBAB hydrate surface probed using a $5 \mu \mathrm{m}$ silica sphere at $-4{ }^{\circ} \mathrm{C}$ in undecane. Spring constant of cantilever: $1.19 \mathrm{~N} / \mathrm{m}$. Right: the corresponding configurations of sphere-surface interactions; arrows indicate travel directions of the cantilever holder relative to the surface. The drawing is not to the scale.

features. In Figure 2 we show only a force curve recorded in undecane as an example.

The jump-in and pull-off forces are indicators of interfacial interactions. We determined the mean values of the jump-in and pull-off forces from force curves recorded in air and in undecane (Figure 3). The results presented for each fluid were

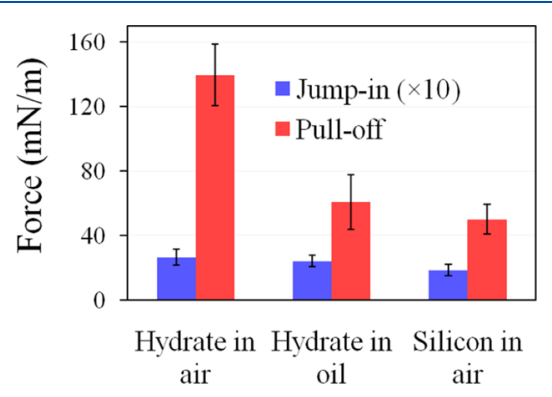

Figure 3. Jump-in and pull-off forces between a silica sphere and a TBAB hydrate surface or a silica sphere and a silicon surface at $-4{ }^{\circ} \mathrm{C}$. The jump-in force is scaled-up by a factor of ten to fit the graph.

extracted from $\sim 30$ force curves. Forces are presented in a normalised unit, $\mathrm{mN} / \mathrm{m}$ by dividing the force by the sphere diameter. In air, the jump-in and pull-off forces of the hydrate surface were $2.6 \pm 0.5$ and $139 \pm 19 \mathrm{mN} / \mathrm{m}$, respectively. In undecane, the measured force decreased to $2.4 \pm 0.3$ and $61 \pm$ $17 \mathrm{mN} / \mathrm{m}$, respectively. As a reference, the jump-in and pull-off forces for interactions between a silica sphere and a silicon wafer (with a natural oxide layer) in air were measured to be $1.8 \pm 0.3$ and $50 \pm 9 \mathrm{mN} / \mathrm{m}$, respectively. The forces on the hydrate surface are larger than the respective forces on the silicon surface. The adhesive force of the hydrate surface in air is almost three times stronger than that of the silicon surface (discussion later).

When looking more closely to the approaching force curves (Figure 4) on TBAB hydrates in air (a) and in undecane (b), they are almost identical except the increased number of data points in (b). We attribute this apparent difference to the higher viscosity that the cantilever feels in the oil medium. The force curves all display well-defined jump-to-contact events. In air, we measured a jump-in distance of $15.9 \pm 2.4 \mathrm{~nm}$ for the spherelairlhydrate. This notation indicates a silica sphere interacting with a hydrate surface across air. For the spherel oillhydrate system, we measured a jump-in distance of $13.4 \pm$ $1.6 \mathrm{~nm}$. In the literature, such a long jump-in distance is explained by capillary forces caused by moisture condensation 

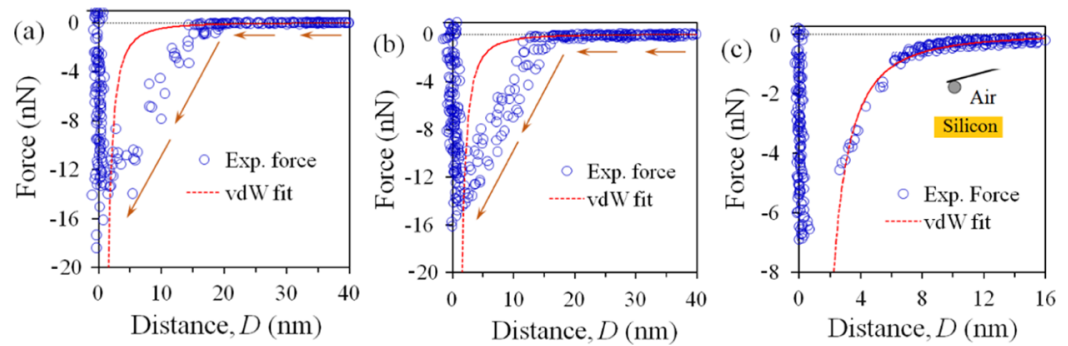

Figure 4. Attractive force curves for interactions between a silica sphere and TBAB hydrate surface in air (a) and in undecane oil (b). Interactions between a silica sphere and silicon wafer (c) for reference. In each graph, results from ten force curves are plotted on top of each other. The spring constant of the cantilever was $1.19 \mathrm{~N} / \mathrm{m}$. The red dashed lines are the best fits of the experimental forces with the vdW theory using eq 1 . The horizontal dotted lines indicate zero force.

in the confined space between the sphere and the surface. ${ }^{39-41}$ This explanation cannot be applied in our case as we measured the forces in undecane (Figure 4b) where moisture condensation could not occur. On silicon wafers, no such long jump-in events were observed (Figure 4c).

We interpret the jump-in behavior of the sphere on the hydrate surfaces as an indication of surface premelting of the hydrates. The jump-in is explained as a jump through a quasiliquid layer (QLL) on the hydrate surface. Fundamentally, surface premelting originates from discontinuity of the structure at solid surfaces. Ice is a specific example of surface premelting. ${ }^{42-45}$ The water molecules in the bulk of the ice form approximately four hydrogen bonds to their nearest neighbours and construct an open $\mathrm{H}$-bonded structure. However, this structure is broken on the surface of ice. ${ }^{42,46}$ The breaking of hydrogen bonds leads to an energetic penalty. ${ }^{46}$ "The energetic loss is balanced by an entropic gain from melting of the surface". ${ }^{46}$ Because ice and hydrates share similar water-based $\mathrm{H}$-bonded structures, we used the wellestablished physics of ice to interpret our results.

Accordingly, the jump-to contact starts when the sphere touches the QLL and stops when the sphere hits the hard crystalline hydrate layer underneath (Figure 5). The same

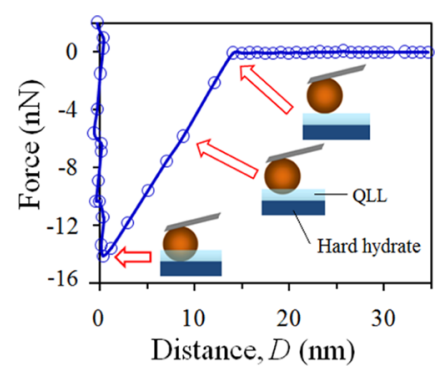

Figure 5. Typical approaching force curve between a $\mathrm{TBAB}$ hydrate surface and a silica sphere in undecane. The insets show free-hand illustrations of a QLL at the hydrate surface. The whole jump-to contact distances are $15.9 \pm 2.4$ and $13.4 \pm 1.6 \mathrm{~nm}$ for spherelairl hydrate and sphereloillhydrate, respectively. The drawings are not to the scale.

formalism was previously used to analyse the QLL on ice ${ }^{43}$ or to interpret the thickness of solid-supported thin liquid films. ${ }^{47,48}$ Thus, we attribute the jump-in to capillary forces originating from the premelting of the hydrate surface. This origin of capillary forces is different in nature from capillary condensation in a humid environment as reported widely in the literature. $^{39-41}$
In a further stage, we fit the approaching force curves with the nonretarded vdW theory ${ }^{49}$ to assess the contributions of vdW interactions. The vdW force between a sphere and a planar surface across a medium can be approximated by ${ }^{49}$

$$
F_{\mathrm{vdW}}=-\frac{A_{132} R}{6 D^{2}}
$$

Here, $A_{132}$ is the Hamaker constant of material 1 interacting with material 2 across material $3, R$ is the radius of the sphere and $D$ is the distance. ${ }^{49}$ We used the combinations silicalairl hydrate, silicaloillhydrate, and silicalairlsilicon.

The force between the silica sphere and silicon surface fits the vdW theory well (Figure 4c). From the fit, we extracted $A_{132}=0.96 \times 10^{-19} \mathrm{~J}$ for silicalairlsilicon. We compared this value with the theoretical Hamaker constant calculated by

$$
A_{132}=\left(\sqrt{A_{11}}-\sqrt{A_{33}}\right) \times\left(\sqrt{A_{22}}-\sqrt{A_{33}}\right)
$$

Here, $A_{11}, A_{22}$, and $A_{33}$ are the Hamaker constants of the sphere material, the surface material, and the medium, respectively. ${ }^{23,50}$ For the silicalairlsilicon, we have: $A_{11}=0.65$ $\times 10^{-19} \mathrm{~J}, A_{22}=2.0 \times 10^{-19} \mathrm{~J}$, and $A_{33}=0.0$, refs. ${ }^{23,50}$ Hence, the theoretical Hamaker constant of the system is $A_{132}=1.14$ $\times 10^{-19} \mathrm{~J}$, which is in agreement with the experimental value above.

Unlike the silicalair|silicon case, the interactions between TBAB hydrate surfaces and silica spheres cannot be fitted by the vdW model (Figure 4). This result further proves that the attraction at hydrate surfaces is dominated by capillary forces between the sphere and the QLL. However, vdW forces may still influence the jump-in distance on hydrates in two ways. First, a minor part of the jump-in could be caused by the vdW force. Second, not only does the vdW force cause a jump of the sphere, but the QLL is also attracted by the approaching sphere and bulges up. This leads to the formation of a meniscus even before the sphere reaches the initial position of the QLL. ${ }^{47,48}$ With the Hamaker constant $A_{132}$ and the spring constant $k$ of our cantilever, we expect a jump-in at distance $D$ to be given by

$$
\frac{\mathrm{d} F_{\mathrm{vdW}}}{\mathrm{d} D}=\frac{A_{132} R}{3 D^{3}}=k
$$

In order to evaluate the contribution of vdW forces to the jump-in distance using eq 3 , we first need to calculate $A_{132}$. To do so, we calculated $A_{22}$ for the hydrate using the Lifshitz$\mathrm{vdW}$ theory following eq 4 , refs. ${ }^{23,50}$ 


$$
A_{22} \cong \frac{3}{4} k_{\mathrm{B}} T\left(\frac{\varepsilon_{2}-1}{\varepsilon_{2}+1}\right)^{2}+\frac{3 h \nu_{\mathrm{e}}}{16 \sqrt{2}} \frac{\left(n_{2}{ }^{2}-1\right)^{2}}{\left(n_{2}{ }^{2}+1\right)^{3 / 2}}
$$

Here, $k_{\mathrm{B}}, h$, and $T$ are the Boltzmann constant, Planck constant, and temperature. The $\varepsilon_{2}=3.3$ and $n_{2}=1.83$ are the relative permittivity and the refractive index of the hydrate, respectively. ${ }^{51} \nu_{\mathrm{e}}=3 \times 10^{15} \mathrm{~Hz}$ is the typical absorption frequency. Substitution of these values into eq 4 gives $A_{22}=$ $3.15 \times 10^{-19} \mathrm{~J}$.

For silicalairlhydrate, substitution of known values into eq 2 gives $A_{132}=1.39 \times 10^{-19} \mathrm{~J}$. Then, from eq 3 with $k=1.19 \mathrm{~N} /$ $\mathrm{m}$ and $R=2.5 \times 10^{-6} \mathrm{~m}$, we have $D \approx 4.5 \mathrm{~nm}$. Thus, the sphere should jump onto the QLL at a distance of $\sim 4.5 \mathrm{~nm}$. Then capillary forces take over immediately so that the whole jump-in shown in Figure 4a is a combination of a vdW-driven jump in air $(\sim 4.5 \mathrm{~nm})$ and a capillary-driven jump through the QLL $(16-4.5=11.5 \mathrm{~nm})$. The latter $(11.5 \mathrm{~nm})$ indicates the thickness of the QLL on the hydrate surface in air (Figure 6a).

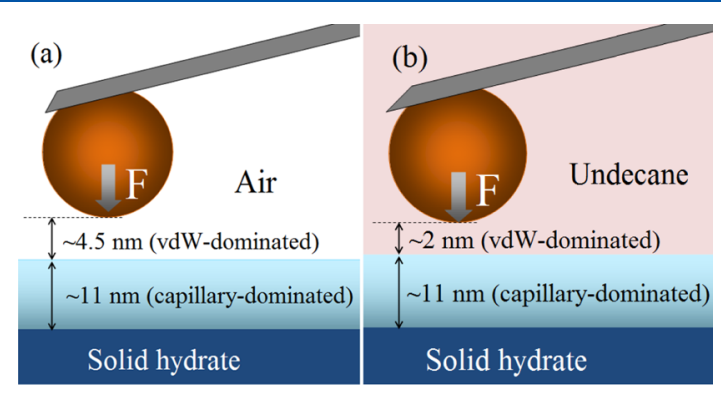

Figure 6. Illustration of the QLL and origins of attractive forces at TBAB hydrate surfaces. The drawings are not to the scale.

For silicaloillhydrate, the Hamaker constant of undecane is $A_{33} \approx 0.50 \times 10^{-19} \mathrm{~J}$, ref 50 . With eq 2 we obtain $A_{132}=0.10 \times$ $10^{-19} \mathrm{~J}$. Then, from eq 3 we have $D \approx 2 \mathrm{~nm}$. The whole jumpin shown in Figure $4 \mathrm{~b}$ should combine a vdW-driven jump in undecane $(\sim 2 \mathrm{~nm})$ and a capillary-driven jump through the $\operatorname{QLL}(13-2=11 \mathrm{~nm})$. The latter $(11 \mathrm{~nm})$ indicates the thickness of the QLL on the hydrate surface in undecane (Figure 6b). Now we can determine that the QLL of hydrates has a similar thickness in air and in undecane, which is around $11 \mathrm{~nm}$ at $-4{ }^{\circ} \mathrm{C}$. This value is comparable with the reported thickness of QLL on ice surfaces. ${ }^{43,45,52}$ Such agreement is logical given that skeletons of both hydrates and ice are constructed on the basics of $\mathrm{H}$-bonded water structures.

Our findings on the premelting of the hydrate surface and its effect on the surface force are of fundamental importance. It settles the dispute as to whether hydrate surfaces are "dry" or "wet". ${ }^{53}$ We can demonstrate that TBAB hydrate surfaces are "wet". Moreover, the strong attraction between hydrates explains the agglomeration of suspended hydrates in flow lines and explains the origin of the flow blockage. The consequences of these findings are that maintaining a stable flow will require technical measures for reducing attraction and/or adding long-range repulsive surface forces, that is, to avoid the agglomeration of hydrate particles. In principle, these technical measures can be achieved by using surface-active agents, or by the appropriate coating of pipe walls. In addition, the thickness of the QLL is a new quantitative parameter that is crucial for modeling interfacial interactions of hydrates. In future, scientists will have to add this parameter to existing models in order to obtain accurate descriptions of hydrate interactions. Both the modeling and prediction of hydrate agglomerations are profoundly important factors for efficient flow assurance. ${ }^{8,53}$

\section{CONCLUSIONS AND REMARKS}

By means of atomic force microscopy, we measured the interfacial forces between a TBAB semi-clathrate hydrate surface and a silica microsphere. We revealed the occurrence of interfacial premelting of $\mathrm{TBAB}$ semi-clathrate hydrate. We demonstrated that the QLL resulting from premelting is crucial to the interfacial interactions of the hydrate. The vdW forces are relevant only prior to the contact of the sphere to the QLL. After contact the capillary formation dominates the surface force of the hydrate. We introduced a new parameter to the hydrate literature, that is, the thickness of the QLL. This parameter is essential for modeling interfacial interactions and agglomerations of hydrates. We have established insights into microscopic-scaled and experiment-based understanding of a semi-clathrate hydrate surface and its applications in the environment and in industry. The scope for future research is substantial. This study has focused primarily on TBAB semiclathrate hydrate and, therefore, similar investigation into other hydrates will be of considerable value and significance. The studies into the effects of surface-active agents on surface forces of hydrates will also be an attractive direction.

\section{ASSOCIATED CONTENT}

\section{Supporting Information}

The Supporting Information is available free of charge on the ACS Publications website at DOI: 10.1021/acs.jpcc.9b06376.

Importance of temperature and humidity controls in the measurements; the preparation of hydrate samples (PDF)

\section{AUTHOR INFORMATION}

\section{Corresponding Authors}

*E-mail: nguyenn@mpip-mainz.mpg.de (N.N.N.).

*E-mail: butt@mpip-mainz.mpg.de (H.-J.B.).

ORCID

Ngoc N. Nguyen: 0000-0002-0999-1176

Rüdiger Berger: 0000-0002-4084-0675

Hans-Jürgen Butt: 0000-0001-5391-2618

Notes

The authors declare no competing financial interest.

\section{ACKNOWLEDGMENTS}

N.N.N. gratefully acknowledges the Alexander von Humboldt $(\mathrm{AvH})$ Foundation for his AvH Fellowship for Postdoctoral Researchers (Fellowship number: VNM 1200537 HFST-P). We would like to thank Dr. Michael Kappl, Uwe Rietzler, Helma Burg, and Andreas Hanewald for their technical assistance in the experimental setups. We thank Prof. Dr. Markus Mezger for helpful discussions on surface premelting phenomena.

\section{REFERENCES}

(1) Thakur, N. K.; Rajput, S. Exploration of Gas Hydrates: Geophysical Techniques; Springer-Verlag: Berlin, 2011.

(2) Koh, C. A.; Sloan, E. D.; Sum, A. K.; Wu, D. T. In Annual Review of Chemical and Biomolecular Engineering; Prausnitz, J. M., Ed.; Annual Reviews: Palo Alto, 2011; Vol. 2, pp 237-257. 
(3) Sloan, E. D. Fundamental Principles and Applications of Natural Gas Hydrates. Nature 2003, 426, 353-359.

(4) Giavarini, C.; Hester, K. Gas Hydrates: Immense Energy Potential and Environmental Challenges; Springer: London, 2011.

(5) Bohrmann, G.; Torres, M. E. In Marine Geochemistry; Schulz, H. D., Zabel, M., Eds.; Springer: Berlin, Heidelberg, 2006; pp 481-512.

(6) Loveday, J. S.; Nelmes, R. J. High-Pressure Gas Hydrates. Phys. Chem. Chem. Phys. 2008, 10, 937-950.

(7) Hammerschmidt, E. G. Formation of Gas Hydrates in Natural Gas Transmission Lines. Ind. Eng. Chem. 1934, 26, 851-855.

(8) Aman, Z. M.; Koh, C. A. Interfacial phenomena in gas hydrate systems. Chem. Soc. Rev. 2016, 45, 1678-1690.

(9) Wang, W.; Bray, C. L.; Adams, D. J.; Cooper, A. I. Methane Storage in Dry Water Gas Hydrates. J. Am. Chem. Soc. 2008, 130, 11608-11609.

(10) Casco, M. E.; Silvestre-Albero, J.; Ramirez-Cuesta, A. J.; Rey, F.; Jorda, J. L.; Bansode, A.; Urakawa, A.; Peral, I.; MartinezEscandell, M.; Kaneko, K.; et al. Methane Hydrate Formation in Confined Nanospace Can Surpass Nature. Nat. Commun. 2015, 6, 6432.

(11) Nguyen, N. N.; Nguyen, A. V.; Nguyen, K. T.; Rintoul, L.; Dang, L. X. Unexpected Inhibition of $\mathrm{CO} 2$ Gas Hydrate Formation in Dilute TBAB Solutions and the Critical Role of Interfacial Water Structure. Fuel 2016, 185, 517-523.

(12) Lee, H.; Lee, J.-w.; Kim, D. Y.; Park, J.; Seo, Y.-T.; Zeng, H.; Moudrakovski, I. L.; Ratcliffe, C. I.; Ripmeester, J. A. Tuning Clathrate Hydrates for Hydrogen Storage. Nature 2005, 434, 743746.

(13) Lee, H. J.; Lee, J. D.; Linga, P.; Englezos, P.; Kim, Y. S.; Lee, M. S.; Kim, Y. D. Gas Hydrate Formation Process for Pre-combustion Capture of Carbon Dioxide. Energy 2010, 35, 2729-2733.

(14) Kang, S.-P.; Lee, H. Recovery of CO2from Flue Gas Using Gas Hydrate: Thermodynamic Verification through Phase Equilibrium Measurements. Environ. Sci. Technol. 2000, 34, 4397-4400.

(15) Nguyen, N. N.; Nguyen, A. V. The Dual Effect of Sodium Halides on the Formation of Methane Gas Hydrate. Fuel 2015, 156, $87-95$.

(16) Nguyen, N. N.; Nguyen, A. V.; Dang, L. X. The Inhibition of Methane Hydrate Formation by Water Alignment underneath Surface Adsorption of Surfactants. Fuel 2017, 197, 488-496.

(17) Tohidi, B.; Yang, J.; Salehabadi, M.; Anderson, R.; Chapoy, A. CO2Hydrates Could Provide Secondary Safety Factor in Subsurface Sequestration of CO2. Environ. Sci. Technol. 2010, 44, 1509-1514.

(18) Park, Y.; Kim, D.-Y.; Lee, J.-W.; Huh, D.-G.; Park, K.-P.; Lee, J.; Lee, H. Sequestering Carbon Dioxide into Complex Structures of Naturally Occurring Gas Hydrates. Proc. Natl. Acad. Sci. U.S.A. 2006, 103, 12690-12694.

(19) Nakajima, M.; Ohmura, R.; Mori, Y. H. Clathrate Hydrate Formation from Cyclopentane-in-Water Emulsions. Ind. Eng. Chem. Res. 2008, 47, 8933-8939.

(20) Ogawa, T.; Ito, T.; Watanabe, K.; Tahara, K.-i.; Hiraoka, R.; Ochiai, J.-i.; Ohmura, R.; Mori, Y. H. Development of a Novel Hydrate-Based Refrigeration System: A Preliminary Overview. Appl. Therm. Eng. 2006, 26, 2157-2167.

(21) Cha, J.-H.; Seol, Y. Increasing Gas Hydrate Formation Temperature for Desalination of High Salinity Produced Water with Secondary Guests. ACS Sustainable Chem. Eng. 2013, 1, 1218-1224.

(22) Xu, H.; Khan, M. N.; Peters, C. J.; Sloan, E. D.; Koh, C. A. Hydrate-Based Desalination Using Cyclopentane Hydrates at Atmospheric Pressure. J. Chem. Eng. Data 2018, 63, 1081-1087.

(23) Butt, H.-J.; Kappl, M. Surface and Interfacial Forces; Wiley-VCH Verlag GmbH \& Co; Weinheim, 2010.

(24) Mokhatab, S.; Wilkens, R. J.; Leontaritis, K. J. A Review of Strategies for Solving Gas-Hydrate Problems in Subsea Pipelines. Energy Sources, Part A 2007, 29, 39-45.

(25) Kelland, M. A. History of the Development of Low Dosage Hydrate Inhibitors. Energy Fuels 2006, 20, 825-847.
(26) Nguyen, N. N.; Nguyen, A. V. Hydrophobic Effect on Gas Hydrate Formation in the Presence of Additives. Energy Fuels 2017, 31, 10311-10323.

(27) Aman, Z. M.; Leith, W. J.; Grasso, G. A.; Sloan, E. D.; Sum, A. K.; Koh, C. A. Adhesion Force between Cyclopentane Hydrate and Mineral Surfaces. Langmuir 2013, 29, 15551-15557.

(28) Aman, Z. M.; Sloan, E. D.; Sum, A. K.; Koh, C. A. Adhesion Force Interactions between Cyclopentane Hydrate and Physically and Chemically Modified Surfaces. Phys. Chem. Chem. Phys. 2014, 16, 25121-25128.

(29) Liu, C.; Li, M.; Chen, L.; Li, Y.; Zheng, S.; Han, G. Experimental Investigation on the Interaction Forces between Clathrate Hydrate Particles in the Presence of a Water Bridge. Energy Fuels 2017, 31, 4981-4988.

(30) Smith, J. D.; Meuler, A. J.; Bralower, H. L.; Venkatesan, R.; Subramanian, S.; Cohen, R. E.; McKinley, G. H.; Varanasi, K. K. Hydrate-Phobic Surfaces: Fundamental Studies in Clathrate Hydrate Adhesion Reduction. Phys. Chem. Chem. Phys. 2012, 14, 6013-6020.

(31) Aman, Z. M.; Brown, E. P.; Sloan, E. D.; Sum, A. K.; Koh, C. A. Interfacial Mechanisms Governing Cyclopentane Clathrate Hydrate Adhesion/Cohesion. Phys. Chem. Chem. Phys. 2011, 13, 1979619806.

(32) Song, J. H.; Couzis, A.; Lee, J. W. Investigation of Macroscopic Interfacial Dynamics between Clathrate Hydrates and Surfactant Solutions. Langmuir 2010, 26, 18119-18124.

(33) Oyama, H.; Shimada, W.; Ebinuma, T.; Kamata, Y.; Takeya, S.; Uchida, T.; Nagao, J.; Narita, H. Phase Diagram, Latent Heat, and Specific Heat of TBAB Semiclathrate Hydrate Crystals. Fluid Phase Equilib. 2005, 234, 131-135.

(34) Makino, T.; Sugahara, T.; Ohgaki, K. Stability Boundaries of Tetrahydrofuran + Water System. J. Chem. Eng. Data 2005, 50, 20582060.

(35) Machida, H.; Sugahara, T.; Hirasawa, I. Memory Effect in Tetra-n-butyl Ammonium Bromide Semiclathrate Hydrate Reformation: the Existence of Solution Structures after Hydrate Decomposition. CrystEngComm 2018, 20, 3328-3334.

(36) Warrier, P.; Khan, M. N.; Srivastava, V.; Maupin, C. M.; Koh, C. A. Overview: Nucleation of Clathrate Hydrates. J. Chem. Phys. 2016, 145, 211705.

(37) Maeda, N.; Wells, D.; Becker, N. C.; Hartley, P. G.; Wilson, P. W.; Haymet, A. D. J.; Kozielski, K. A. Development of a High Pressure Automated Lag Time Apparatus for Experimental Studies and Statistical Analyses of Nucleation and Growth of Gas Hydrates. Rev. Sci. Instrum. 2011, 82, 065109.

(38) Gan, Y. Invited Review Article: A Review of Techniques for Attaching Micro- and Nanoparticles to a Probe's Tip for Surface Force and Near-Field Optical Measurements. Rev. Sci. Instrum. 2007, 78,081101 .

(39) Badal Tejedor, M.; Nordgren, N.; Schuleit, M.; MillqvistFureby, A.; Rutland, M. W. AFM Colloidal Probe Measurements Implicate Capillary Condensation in Punch-Particle Surface Interactions during Tableting. Langmuir 2017, 33, 13180-13188.

(40) Sprakel, J.; Besseling, N. A. M.; Leermakers, F. A. M.; Cohen Stuart, M. A. Equilibrium Capillary Forces with Atomic Force Microscopy. Phys. Rev. Lett. 2007, 99, 104504.

(41) Vitorino, M. V.; Vieira, A.; Marques, C. A.; Rodrigues, M. S. Direct Measurement of the Capillary Condensation Time of a Water Nanobridge. Sci. Rep. 2018, 8, 13848.

(42) Li, Y.; Somorjai, G. A. Surface Premelting of Ice. J. Phys. Chem. C 2007, 111, 9631-9637.

(43) Döppenschmidt, A.; Butt, H.-J. Measuring the Thickness of the Liquid-like Layer on Ice Surfaces with Atomic Force Microscopy. Langmuir 2000, 16, 6709-6714.

(44) Nagata, Y.; Hama, T.; Backus, E. H. G.; Mezger, M.; Bonn, D.; Bonn, M.; Sazaki, G. The Surface of Ice under Equilibrium and Nonequilibrium Conditions. Acc. Chem. Res. 2019, 52, 1006-1015.

(45) Slater, B.; Michaelides, A. Surface Premelting of Water Ice. Nat. Rev. Chem. 2019, 3, 172-188. 
(46) Limmer, D. T. Closer Look at the Surface of Ice. Proc. Natl. Acad. Sci. U.S.A. 2016, 113, 12347-12349.

(47) Ally, J.; Vittorias, E.; Amirfazli, A.; Kappl, M.; Bonaccurso, E.; McNamee, C. E.; Butt, H.-J. Interaction of a Microsphere with a Solid-Supported Liquid Film. Langmuir 2010, 26, 11797-11803.

(48) Schellenberger, F.; Papadopoulos, P.; Kappl, M.; Weber, S. A. L.; Vollmer, D.; Butt, H.-J. Detaching Microparticles from a Liquid Surface. Phys. Rev. Lett. 2018, 121, 048002.

(49) Butt, H.-J.; Cappella, B.; Kappl, M. Force Measurements with the Atomic Force Microscope: Technique, Interpretation and Applications. Surf. Sci. Rep. 2005, 59, 1-152.

(50) Israelachvili, J. N. In Intermolecular and Surface Forces, 3rd ed.; Israelachvili, J. N., Ed.; Academic Press: San Diego, 2011; pp 253289.

(51) Ahn, Y. H.; Kang, H.; Koh, D. Y.; Lee, H. Terahertz TimeDomain Spectroscopy on Semi-Clathrate Hydrates: Optical Properties Based on Different Structures. Int. J. Chem. Environ. Eng. 2014, 5, 271-276.

(52) Murata, K.-i.; Asakawa, H.; Nagashima, K.; Furukawa, Y.; Sazaki, G. In situ Determination of Surface Tension-to-Shear Viscosity Ratio for Quasiliquid Layers on Ice Crystal Surfaces. Phys. Rev. Lett. 2015, 115, 256103.

(53) Maeda, N. Is the Surface of Gas Hydrates Dry? Energies 2015, 8,5361 . 